\title{
Natural Sciences are a Natural History
}

\author{
Shozo OHMORI \\ Tokyo University
}

In those days, when we say "Natural History" we usually mean a pleasant and pastoral corner of the natural science, somewhere near the playground of sunburned nature lovers. But a natural historian himslf would be puzzled if we tell him he is doing history. Much more the muclear physicists who are trying hard to think out a theoretical envelope to control ever overpopulating elementary particles, or biochemists pursuing the microscopic labyrinth between DNA and protein production are certainly astonished and probably would get angry if we call them historians of Nature. But, though they certainly live and work in the metropolis of the science, they are still working at the Nature just as well as their rural fellow scientists are. And when they work at This Nature, they inevitably have to work as historians of This Nature. And they are historians. The reason is discouragingly simple. It is because there is only one nature, The Nature.

However it is not at all a simple matter to draw a picture of the science as a history of the Nature which can pass as a full-sized portrait of it. Instead, in the followings, I only wish to try a sketchy dessin, hoping the unwillingly introduced déformé there would not blur the original scenery but rather help to make it's fundamental outlines clearly visible.

\section{Natural law}

There are varieties of natural laws. At it's simplest end we have simple generalizations like "all crows are black" or "in all the equilibrium states of gas, $\mathrm{PV} / \mathrm{T}$ is approximately constant" etc. Obviously those laws are packet statements wraping a great number of individual observations in the past, plus extended predictions for indenifite number of future individual cases. That is, what this kind of law expresses are actual and predictive reports of individual events or states of this world taking place at certain times and places. They are historical reports and historical predictions. As such, though they are easy to grasp, but impossible to be discovered without a tremendous accumulation of observations. It is true that we do not seem to have their exact counterparts in human history. This is beccause we are never interested in a quite boring observation such as Caesar saw Rubicon with his two eyes or Stalin used to wlak on his two legs. So we don't have generalization laws in human history like "most men are binocular and biped". 
Instead if Caesar became blind on the bank of Rubicon or Stalin started to walk on all fours in 1944, we make them very important historical records. The difference is only a matter of importance relative to our interest and concern, not a matter of historicality.

Still one may object against me by saying: I have totally misunderstood the real nature of natural laws. Natural laws are not mere records of what happened and what will happen even when they are simple generalizations. Laws are not historical records of how things were and will be, but laws state how things must be whenever they happen. Laws are laws things must obey or must accord to. In short, I am a Humean anachronism.

I am quite ready to put aside the "black crows" case which is too obviously an obvious exception to the above objection. And if I ask the objector what it is to make the air obey the Boyle Charles's law, he is supposed to answer me back by deducing it from more fundamental laws of thermodynamics or statistical mechanics. Then it is a natural course to ask same questions about those laws. After a series of these question and answer in several repeatances, we will find ourselves at the end of the game. There stand the basic laws of physics.

However, man can no longer appeal to another law in order to establish the law-ness of basic laws. They must stand on their own foot. But do they stand to command the world to follow them? Let us take the Newton's equation of motion, as a standing example in classical physics. And let us assume, for the sake of simplicity, that all forces appearing in it are either given as external forces or uniquely calculatable in terms of the kinetic and other properties (e.g. electromagnetic) of moving bodies. Now we ask, what is the function of Newton's equation? Can it tell us when and where of the next eclipse? Can it show us we make a round trip around the sun in 365 days 6 hours and so? Certainly not. Let it alone, it has nothing to tell about any concrete fact of the world. Only after it is supplied with particular data called initial and boundary conditions, it begins to speak on how the world actually goes.

Thus Newton's equation is nothing but an apparatus waiting concrete reports of this world put into it that it may put out another concrete reports. The form of differential equation is just a right fit for this function. Differential equations are a sort of prescribed documentary forms with several empty blank spaces expecting to be filled out. As they are, they do not inform us of any particular state of affairs. They are unfilled papers, or we may say they are half-made documents. Once we fill out all blanks with particular data (integral constants) then we get fully written out papers.

What those completed papers are about? If the data we put into the apparatus were some actual reports of the world, then the produced papers are supposed to be some true statements of the world too. If we find them not, we 
either reexamine the input data or abolish the apparatus itself. This clearly shows that natural laws are devices neatly designed for the purpose of producing indenifitely large number of particular reports of the world, or special forms of writing down all of them with the aide of empty-elft blanks. Of course we can put any arbitary data into it instead of actual observations, and let the data-producing machine go. However, unless it is intended as a strategy for the purpose of somehow reaching the ture statements of the world (for instance, in order to test a hypothesis), it is not more than the idling or exercise of the apparatus. No one would dare to say he is thus calculating possible states of a possible world. Physics is not logic.

Thus we come to see that natural laws are not some abstractions common to all phenomena in certain domain, as said from time to time. On the contrary, they are huge comprehensive descriptions including all particular descriptions in the domain without leaving any bit of their particular details out. This is, at least, the true picture of natural laws in use, not in stock. And this is also the reason why we can deduce particular statements from a law. Unless a consequence is already contained in premises, we cannot deduce it from the latter, this is a fundamental metatheorem of logic, though a very informal one I admit.

Now if we ask straightfowardly what makes the world obey Newton's law (approximately), I think there is no answer other than to say that the world proceeds as a matter of fact in accordance with particular descriptions produced from it. And because Newton's law itself is a comprehensive set of particular descriptions as shown above, this answer clearly amounts to a tautological observation (incidentally, this is the bare kernel of the so-has-been-called "explanation by law"). What remains substantial is again a general fact that this world proceeds as a mattar of fact in such a way as expressed by Newton's law. If we ask why so, it is like asking "why it is sky?" looking up the sky. The question disappears into the vacuum because it is vacuous.

Natural laws in use are descriptions of the world, including betted or anticipated descriptions for the future. And there is only one world in which we live and of which science studys. And this world is a temporal world. It changes in time, it proceeds in time. It has it's past, it's present and it's future. It's past is eternally lost into the past, can never be recovered. It's future has never been lived, and never will be. Everything, every state, every phenomenon in this world was just once, is just once and will be just once. Everything there has it's own particular date. Then, if we want to describe this world, we do not have any other way than to record what happened when and where and how and to anticipate what will happen. This mode of description is just the thing I want to call "historical". In this sense, all scientific work is historical, and cannot be otherwise, cannot be anything else.

Most scientists, with the exception of astronomist, geologist, evolutionist and 
possibly psychonanalyst, seem not to care for particular dates at all. They regard their particular observation or experiment with a particular date nothing but an instance of a general law which they are aiming at and which, as such, has no date. But they usually forget that their timeless laws themselves are nothing but special writing forms to write down an infinite set of particular descriptions with particular dates in past, present and future tneses. Again, laws are nothing but the recipe formulae to cook out particular descriptions. Hitorians of human society, on the other hand, (except in the field of economics) can never forget of this, mainly because they have not yet succeeded to find even a single interesting law-like law, and granting they could find one it would be so sensitive to particular descriptions and so ready to revisions that they could never mistake it's real character as a packet of particular descriptions. Human history is, in it's nature, a set of descriptions so poorly organzied that historians cannot get drunken by the spirit of law. In contrast to their soberness, scientists quite often, drunken by their enormous success, easily fall into the fetishism of law.

Natural laws are hitorical laws of This World. Their function in use, their performance at work is no more and no less than to produce particular records of this world, how it was, how it goes and how it will be. Therefore, if the aim of the science is to find laws of this nature, then the target of the science must be to get the unique historical records and will-be records of This unique world.

\section{Atomistic description}

Granted that science is a historical study of this unique world, it has a very peculiar style of writing it's works. Besides scientists want to write their history in deductive organizations by alw-systems (hypothetico deductive systems), as far as possible, they draw their picture of the world in a pointillistic style with colorless, odorless and tasteless vocabulary. Science uses an atomistic brush as it's most fundamental mean. Then, how it can be a description of our live world full of color, smell, taste and sound?

The reason why our science, gradually in the course of it's development, came to take the atomistic description is a deeply philosophical one. This is another thing which scientists are not aware of. They seem to me not so much self-conscious on their own style as painters are, though scientists are in a sense landscape painters in four dimentions.

I think there are only two fundamental styles to describe something spacial. (A) Thing-property description; when expressed in language, the subject-predicate form. (B) State-in-space description, or field description; when expressed in language, the state-existence form. Those are the two and I cannot think of any third on a par with them. They are something very near to Kantian categories, at least as 
fundamental as the latter though I don't believe they are a priori.

Now, it is a quite old story in philosophy that when we want to talk about a changing phenomenon in space and time we inevitably need to have something like the notion of "substance" or "substratum" which stays unchanged through the change and carries the changing properties on it. Although "substance" is patently a notorious metaphysical notion, I think the story is still quite true unless we switch to (B), the field description. Of course first we must shift from metaphysical substance to physical substance, the "thing". A hard minded positivist will raise at once an objection and say: "The physical substance is just as much as metaphysical. Deprived of all it's properties, the "thing" becomes entirely transparant and empty. The truth is that any statement about things is a façon de parler which is translatable into an infinite set of perception statements and only as such it is empirically meaningful. In other words, the only meaningful style is (B), and (A) is reducible, in a very peculiar sense, to (B). Indeed the socalled phenomenalist language is in the style (B)."

I agree with him as I am a hard minded positivist too, at least on this point. But it is an entirely different matter to say that in the style (A) the "thing" is the indispensable notion to carry over the role of "substance". In fact we can meaningfully tell exactly where this or that "thing" is, exactly where it is moving, what shape and color it has and above all, which is the same "thing" which changed it's position, shape, color etc. What we cannot meaningfully tell is, what properties the "thing" has as the "thing-itself". This is simply a simple contradiction. There does not exist anything such as "thing itself" without any property, but a "thing" which has certain properties is nothing but a common thing-desk, or a thing-chair.

I am well aware that this kind of talk is a needlessly pedantic chattering about common desks and chairs, only to irritate common sense. But I needed it because I think here is the junction for the scientific picture to pass the common landscape ahead.

Now let us take a classical example. Looking at a lump of clay constantly changing its shape in a sculptors' hands, we ask what it is that remains same through all those deformations? Of course, it is the lump of clay. However, what does it mean to say the clay remains same when it is changing it's shape? What is the same "thing" between the initial lump and the finished head of Venus? The only answer will be this. If every parts of the initial lump are contained in the Venus and vice versa, we say those two are the same thing, and if every parts can be traced through a metamorphose then we say the whole is kept same through the change. (If translated into Prof. Quine's interesting phrase, two things are same when both are temporal cross-sections of one and same four dimentional thing. - From a Logical Point of View, p. 69) But immediately a question. What 
does it mean to say a part stayed same? Any part as well may change it's shape and volume (by expansion and compression). Then, we have to start talking about a part of a part and again a part of a part of a part. Should we not let it go into an obvious infinite regress, there is only one way out, that is, to stop the regress. And the only way to stop the regress must be to suppose everything is composed of parts which themselves no longer change their shapes and volumes but change only their positions in space. Those parts are unmistakingly something like "atoms", but not quite so yet. So let us call them, for a while, "grains".

I want to stress the fact that the course from the style (A), "Thing-property" description to "grain" description is almost a logical necessity. Once we take the thing-property description, it is inevitable to take the grain description unless we make a U-trurn to the style (B), the filed description, or give ourselves into an infinite regress. I hope this is sufficiently established in the above. Then one may ask "If so, why the ordinary language has not followed this supposedly inevitable course?" Right here is the most fundamental difference between the common sense description and the scientific description. Science wants and demands a completely detailed picture of the world, at least as complete as possible, from the unlimited curiosity of man. This pursuit of detailed description was the very cause of uncovering the "grain" structure built in the "Thing-property" description. On the other hand, in ordinary life we don't need a so much detailed picture of the world, neither we have a demand for it. An excess of details is rather harmful to our common life, as any man will admit. In accordance with this, our ordinary language stops far short of scientific details (at least out of the power of discrimination of our sense organs). In fact the ordinary language is a rather rough-meshed classificatory language. This is the reason why it is quite satisfied not to reach the junction point where the "grain" structure in the ordinary subject-predicate forms inevitably become visible.

In the history of science (that is, in the history of the historical descriptions of the world), no one fails to see that it passed that junction already in 17 th century (at least for me, this is the most dramatic aspect of the so-called scientific revolution), and it has kept pursuing more and more detailed descriptions with more and more vigor up to the present time. However it is also an old fact that long before the science actually passed the junction, the "grain" structure was already discovered in human minds. What philosophers called "atom" in Greece is a "grain" but not vice versa and again is not exactly a modern "atom". But it is certain that their atom was at least something which does not change and remains same except changeing it's spacial location. This is enough to make it a "grain." The detailed circumstances of how they came to discover it, I have to leave to the historians of philosophy. However, I believe that a kind of Gedanken Experiment (we may also call it "analysis") of pursuing spatio-temporal details of changing 
things played one of main roles in it. Especially they rightly paid attention to cases of the concentration and rarefaction. Because those are just the typical cases of change of volume and shape where I think the unavoidability of "grain" is most. easy and clear to be seen. It is interesting to remember that Descartes seems to. admit the grain-ness in those cases within his horror vaci space (Principles, Part II, section 5,6 ), at the same time rejecting the atom-ness or the indivisibility (section 20). Anyway it seems to me that the history of both philosophy and science accords. well to the inevitability of the "grain".

As mentioned above, "grain" is a broader notion than atom. It is necessary and sufficient for a grain to be a part of a thing which does not change it's shape and volume through changes of the thing. As such, a grain is a spacial thing which is identifiable and tracable as a same thing and thus as the only legitimate subject of possible predicates in detailed descriptions. Therefore it can take any shape and size so far as they remain invariant. Remember various fantastic figures of Epiculian or Lucretian atoms dancing in their books, though only in their books and letters. It is purely an empirical matter to find out how big it is and what shape it has in this world described under the style (A). Also grains are, unlike atoms, divisible. The problem of deciding to what extent they are divisible (in other words, how small they are) relative to a set of observations we take up is again a factual matter. A grain in a certain domain of observations may be no longer a grain in other domains. Grains in relatively quiet processes quite naturally tend to stop being grains in more violent processes (with higher energies) and to be replaced by smaller grains, their previous parts. As an obvious fact, we have witnessed the procession of stepwise splitting of grains, - elements (?)-moleculesatoms-nuclei-elementary particles. Whether we now stand at the end, or we are coming near the end, or we are only at the start of the procession, no one knows. But I want to stress one very important fact. This procession into ever smaller grains is at the same time is a procession into ever more detailed descriptions. The pursuit of details and the enlargement of observations, hand in hand, did produce the march to smaller grains.

However, grains are still neither antique nor modern atoms. Atoms usually were and are supposed not to have any sensory properties. They may be conceived as causes of or the basis (whatever it may be) of sensations, but we cannot meaningfully attribute any sensory properties to them except geometrical ones. It has a shape and size, but no color no taste etc. But a grain can have sensory attributes, because all we demand of it is nothing more than the invariance of the shape and the size. Grains of sugar can be white and sweet, whereas atoms of sugar cannot be of any color or taste. For the common sense, any small grain of sugar is just a small bit of sugar however small it might be. But for the science, a cube of sugar is neither sweet nor sour however big it might be. It causes a sense of 
:sweetness when and only when it comes in contact with atoms of a tongue. Then what makes us shift from grains over to atoms?

As explained so far, the notion of "grain" was the inevitable result when we pursued details of the "thing" in the "thing-property" description. Now if we turn to the details of "property", we realize that we are almost forced to form the notion of "atom". This time, the birth of the notion of "atom" is not completely inevitable as it was in the case of "grain". But it is practically inevitable in the sense that we don't know any birth-control method workable to stop it.

First, it is a transparent fact to anyone that sensations are something which depend on our sense organs. Thus sugar tastes diferrently depending on the conditions of my tongue, and it looks in different colors (putting aside the other mind problem for a while as I am now talking in common sense on common psychology) to different persons. This makes it impossible to speak of a same property independent of our sense organs. Secondly, everyman knows that sensory properties are dispositional. Sugar is sweet only in the mouth. People may say, no it is just as much as sweet in a sugar box. But if pressed, they surely must retreat to a dispositional talk or something like the potential sweetness, the sense producing power or the cause of sensation etc. But it is too clear that any of those potentiality, power or cause is not sweet at all. They are not sensory properties. This makes it impossible to speak of a thing's sensory property independent and outside of our sensations.

It is quite natural, or rather practically inevitable for us to be led into the thing-sensation dualism by those two observations above. Things cannot have sensory properties. Snesory properties are somehow caused or produced or paralleled by non-sensory things. Now things are "grains", and must be. Then those "grains" cannot have sensory properties, that is, "grains" must be "atoms."

Thus we now arrived at "atoms" after a little long but straight journey. Our point of departure was the "thing-property" description, the most fundamental statement form in our language. Then we found the notion of "grain" is inherent in this type of description in the domain of spacio-temporal phenomena. The notion "grain" is inherent in the notion of "thing" in space and time. Next we turned to the notion of "property" and found sensory properties not attributable to "grains". So, "grains" and accordingly "things" in "thing-property" descriptions must be "atoms". Thus we can conclude: So far as we stay in the almost inevitable thing-sensation dualism, the notion of "atom" is inherent in the "thingproperty" description. (Caution. Our atoms need not to be a-tomon, as mentioned before.) Therefore, if we push the "thing-property" description into details, it, is almost necessary to take the atomistic description.

However there is left one important problem. In the above, I kept the thingsensation dualism purposely on halfway. But if we push it to the end, it stops 
any longer to be a dualism. Because there cannot be any Lockean distinction between primary and secondary attributes, the shape and size of an atom is no less snesory than color or taste. Then atoms cannot have any sensory properties including shapes and sizes. Atoms are in danger of evaporating into no-thing or shrinking back to metaphysical thing-itself. Here, we seem to have two wayouts. (1) To surrender atoms to the phenomenalism. That is, we admit that the atomistic description is no more than a façon de parler in the phenomenalist language. (2) To endow atoms physicial shapes and sizes, giving up sensory shapes and sizes to the sensation. This amounts to admitting two independent ways of describing our one world, somehow later establihsing (or postulating beforehand a connection or correspondence) between those two descriptions.

However, a shape (accordingly it's size) is a particular set of positions in space, as clearly shown in the analytic geometry where geometrical figures are defined and expressed by a set of points satisfying particular conditions. Therefore, to talk about the shape and size of atoms is nothing but to talk about positions occupied by atoms. Now there is only One space we and scientists live in. Then there cannot be two different kinds of positions in it, one physical the other sensory. There can be a physical shape of a table different from our sensory shape of it (Locke was wrong here). But this difference is a difference of two different sets of positions, in the same space, not in two different spaces. Different shapes are made up of one kind of positions in One space, where we live, see colors and hear sounds. Atoms are of course in this space, they cannot and should not have alibi in this space. Atoms must share this psace with sensory properties. Accordingly their shapes are just of the same kind as the sensory shapes of a table we see in full technicolor.

Therefore, if scientists take, as they in fact do, the wayout (2), they still have to share positions with sensory properties. All they do is to make a distinction between physical and sensory shapes made of positions in the same space. Without thus sharing the space with sensory properties, the atomistic description cannot be even a description of this world.

Thus so far as it stays in this world, the atomisitic description is nothing but the common "thing-property" description only with more details than common. In this point, it has nothing foreign to ordinary descriptions. The only trouble with it is that it cannot describe the world completely. They do not have and cannot have sensory vocabulary. So it's picture necessarily must remain colorless, tasteless, soundless and neither hot nor cold. In it, human bodies move away at a soundless explosion of a colorless volcano. However this is no real trouble at all. Science has prepared dictionary or a manual capable to tell us, given an atomistic picture, how to paint it, how to play a melody with it and so on. And this dictionary is also growing steadily with growing stock of scientific descriptions. It is a job for philosophy to make the nature of the dictionary 
clear, but the job for science is to use it in making historical records of this world.

\section{Eield Description}

The atomistic description is no more than a detailed "thing-property" description. And it is not the sole style of description which science and common sense use in their world description. Science as well as common sense use the other style, the "field" description or "state-in-space" description mentioned previously.

In our experience there are a few domains where we don't necessarily need to adhere to thing-property descriptions and it is rather natural to use field descriptions. Among others, the inner sensations are a fit for them. Instead of "I have a pain in my fingers", we can say "There exists a pain in my fingers". And I think the latter expression, though queer in customary usage, is a more straightfoward copy of the experience. Because in the pain I do not see anything like a "thing" or a substance. There simply is a painful sensation or a painful state. Where? At my fingers. Accordingly it is enough and clear to describe it in the form "A certain state exists in a certain area in space". Same with taste and tactual sensations. Sweet sensation exists on the surface of my tongue. A hot feeling at the tip of the thumb. In general, we can use field descriptions in describing mental experiences localized in the space.

Field descriptions, as such, do not demand anything to sustain through changes of states. When a stomackache increases it's intensity, simply a change of states is taking place in the stomach and there needs to be nothing to remain same through it (Incidentally, I think Kant's distinction of extentional and intentional quatities has it's root here.). Thus field descriptions are not in the proper subject-predicate form, but are in the state-existence form. Therefore they don't have the trouble which the thing-property description had to cope with when pushed to detailed descriptions. Hence the field description is far more transparent and free from troubles compared to the latter. This is one important reason why the common trait of various phenomenalisms is to try to describe not only the inner experiences but the whole world in the field descriptions. In fact, all varieties of phenomenalists languages, most pointedly the sense-data language, are more or less in the filed description style. And their business, a very difficult business, is to translate the thing-property descriptions off into the field descriptions.

Apart from any philosophy, however, scientists carefreely have adopted the field description whenever they thought it appropriate. Sometimes they use the field description overlapping the domain already covered by the thing-property description or the atomistic description like cases of the elasticity or hydrodynamics. But also it had it's own use quite independent of the thing-property descriptions, for instance, in the electromagnetic filed or in the gravitational field. It is interesting to remember that scientists once tried hard to return back to the thing-property 
description by postulating a "thing" - ether - in the electromagnetics. They are born materialists, feel unsecured unless they have "things" in their hands to cling to, while, on the other hand, they have to admit some field in empty space to replace the action at a distance which they hate instinctively.

Those filed descriptions in science is nothing different from the common field description. A scientific field description is no more than a detailed form of a common field description, exactly as an atomistic description was nothing but a detailed form of a common thing-property description. Moreover, field descriptions in science and common sense slide more smoothly into each other compared to the thing-property description just because there can not exist any counterpart of "atom". But the field description in scienc still has a decisively important difference from the common field description, in that the former entirely lacks sensory vocabulary which is the very vocabulary of the latter. An electromagnetic wave of any wave length has no color at all. It produces a sensation of red or blue only when it hits the end of our nervous system or the receptor which itself is again an aggregate of colorless atoms. The reason for the lack of sensory vocabulary in scientific field descriptions is again very simple in contrast to the case of the atomistic description. It is just because there is, from the start, nothing in the electromagnetic or gravitational fields we can see, hear or smell. We see a table or dusts, but we never see the lights which make us see them. Falling down a staircase, people don't see or smell the force pulling down them. Hence the scientific field description cannot have those sensory vocabulary. But of course, as the atomistic description is, it has to share position words with sensory vocabulary. Without this it cannot be a description of this world.

In the history of science, both atomistic and field descriptions played each roles in an intimate cooperation. But in the quantum mechanics we saw the field description taking over it's atomistic friend. This is a struggle between two most fundamental frameworks of description, the "thing-property" and the "statein-space" descriptions. No wonder we got into a deep embarrassment from which we are not yet freed. The well known probability interpretation of the state function is a kind of compromise between them. But I think it cannot overcome the difficulty of the single-case probability, though there might be a wayout such as suggested by Prof. Popper (S. Koelner ed. Observation and Interpretation. 1957). I am rather inclined to switch completely over to field descriptions from the atomistic descriptions. Thus a state function is a pure field description assigning particular complex numbers to continuous positions in space. Of course one may ask about the physical meaning of such complex numbers. But I think if we could have an observational or sensory meaning of field descriptions as a whole, then we need not worry about physical meanings. Of course things here is not so simple at all. I only want to point out that we are witnessing in quantum mechanics and 
thories of elementary particles a real sttuggle between our most fundamental styles to describe this world. I really hope, through this struggle the basic structure of the description of this world will be made visible in the near future.

\section{Theory}

It is too familiar a fact to be noticed attentively that when we see the world around us we see only surfaces of things in it. We see the front of a house but not it's inside. In a house we only see the surfaces of ceilings, walls and furnitures. We can see neither transparent things nor the insides of opaque things. We only see the surfaces of opaque things. Only few people had rare chances to see inside of his skins. In order to see the inside of something we must open it to turn it's inside to outside surface. Before opening we often make a hypothetical description of how it's inside is. This is to posit a dsecription of a small part of the world which we don't have access at that time. Our everyday life depends heavily on those posited descriptions but they are usually not intereting to scientists. However when it comes to the posited descriptions of how it is inside of our earth, our scalp, our nerve cells and our chromosomes, they are called "theories". Roughly speaking, theories are posited descriptions of something which blockades easy access to it.

The sun, moon, planets and stars are too far to approach. So posited descriptions of their temperatures, their ages or their histories are theories. On the other hand, fine structures of things are also very difficult to access. So, posited descriptions of them are patently theories. Here, those theories are at the same time detailed descriptions of common things. In this sense almost the whole of physics, chemistry, biochemistry or biophysics are theories.

We ourlseves and our capacity of observation are extremely small and limited in the world we live in. Nevertheless science as an ideal wants to get a complete description of the world. Then it is inevitable for the science to appeal to a lot of guess works. And those guess works are called theories. The present picture of the world is very far from a complete picture. Most of it are still left unpainted. Scientists have been trying to fill it's blank space cautiously with theories, checking again and again by confronting them to controlled observations. Also They seeked after a more and more detailed picture not only because they pursued details as such but because a detailed picture is always a simpler picture in structure. And a detailed picture is nothing but a guess work called theory.

Thus, it seems to me, there are two main directions in our indispensable use of theories. One direction points toward spatio-temporal areas we have not yet brought into observations or we can never observe. Typical examples are, the socalled dynamo theory of the inside of the earth with an intention to explain the terrestrial magnetism, or the well known evolution theory of plants and animals intended to describe the historical genealogy of creatures in the past we cannot 
observe for ever. The other direction is of course toward details of everything, exemplified by almost any theory of the modern physics, chemistry and molecular biology. Taken together, theories are posited descriptions of science with the aim to paint a complete panoramic picture of the world, while we, as common individuals, can observe only sproadically dispersed narrow parts of it through peep-holes of rough naked eyes and ears.

In a loose sense, science is playing a gigantic picture-puzzle of the world. They are given only a few pieces of it, and their task is not only to complete the whole picture which fits given pieces but also to replace them by far more detailed ones. They are re-painting our common rough picture to make it a picture of the unvierse. However, their picture must fit our observations to the last however limited they are. Otherwise their picture is nothing but a scientific scribbling however well done it may be. Accordingly the basic criterion of right or wrong of scientific theories is how good and exactly they overlap our observational descriptions and how good they fit each others in the whole picture of the world. I think here is the core of the so-called scientific explanation. When a picture painted by a theory exactly overlaps our observational descriptions, the theory explains those observations. The deduction of observation statements from the theory is a mere formal reflection of this overlapping. I think what Prof. Toulmin in his "Philosophy of Science" wanted to stress was this point from a different angle.

On the other hand, a theory cannot be a theory if separated from other theories. A theory is a theory in the whole picture of the world. And this whole picture cannot be a patched work of many independent theories. The world picture is one picture completely welded. So with theories. A local theory is overlapped by a wider theory, and the latter in turn is overlapped by a still wider theory untill we get a completely welded world picture. But what does it mean to be a "wider" theory. It has two meanings. One obvious meaning is to be more comprehensive. In addition to this unexciting one, there is another meaning. That is being more detailed. The theory of muscle cell contraction explains the bending of our arms and legs by overlapping it. But how the muscle cell contract is explained when it is overlapped by a more detailed theory, for instance of Huxley, and again the latter is waiting to be overlapped by a more detailed actomyosin theory on molecular levels. Thus the pursuit of details is again the kernel of the evolution of theories, a more detailed theory replacing it's precursor by overlapping it. In one aspect, theories are products of ever more detailed atomistic and field descriptions. If we want to get a wider theory, we necessarily need a more detailed atomistic and field descriptions.

However the field description is, from the start, in the last details, being a state description in continuous space. We cannot pursue more details over points in 
space. Points or positions are the end or the bottom of details. So, if we pursue details, it is in the atomistic description. In fact, as repeated before, this is what has happened in the history of science.

As stated in preceeding sections, atomistic descriptions lack sensory vocabulary like colors or tastes. What are left for them are no more than geometrical vocabulary, fundamentaly just positions in space and nothing else. More exactly, positions and movements of positions of atoms. in our sense. Other physical properties and states are all defined in terms of this kinetic vocabulary. Masses, forces, electric charges, magnetic vectors, electric currents, spins are all defined, though in an enormously complicated architecture, in terms of movements of atoms. Although I am afriad I am not capable to prove this convincingly if I have enough room here which I don't have now, it seems to me this is a necessary consequence from the very nature of the atomistic description. Deprived of sensory properties, the atomistic description inevitably stay in kinetic descriptions.

If I were right in this, theories also must be kinetic descriptions of atoms on a certain level, so far as they stay in the "thing-property" style of description. Then the scientific picture of the world is nothing but a gigantic picture of the movements of those atoms. As such, it is a wonderfully elegant and simple picture, if not artistically but at least intellectually. However it is utturly an impossible task to paint actually even a sketch of the whole universe with all billions of scientists. Therefore we have to be satisfied with an apparatus capable to produce any part of the picture if we supply it only a part of that part to be produced. This apparatus is nothing but the thing called basic laws or basic theories. Basic theories as well as basic laws are the description apparatus to paint the actual picture of the world.

Here I ended up with a rough sketch of the function of our science. Hoping I am roughly correct in it, I want to say: Science is the detailed description of this world we are living in. The style of description it adopts, the atomistic and field description, is an almost inevitable extention into details of the common style we use in everyday life. And thoeries and laws of science are tools or apparatus to describe our immense world with the very limited power of human beings. And what does science describe? There is nothing to be described else than this world rolling in time from the past to the future. Then Science cannot be other than the history of this unique world. Natural sciences are a Natural History in full sense. 